\title{
EL PERSPECTIVISMO EN LOS NUEVOS ENSAYOS SOBRE EL ENTENDIMIENTO HUMANO DE LEIBNIZ
}

\author{
Manuel Sánchez Rodríguez'
}

\begin{abstract}
RESUMEN: En este artículo se analiza la concepción perspectivista del conocimiento, tal como es expuesta por Leibniz en los Nuevos Ensayos, como uno de los aspectos que marca la originalidad de este pensador en la Ilustración racionalista. Se defenderá que esta concepción se sustenta en dos tesis. En primer lugar, Leibniz sostiene que todo conocimiento humano es esencialmente perspectivista, pero esto no impide sostener, contra el escepticismo, que en cada caso las diferentes perspectivas ofrecen un conocimiento diverso de un mismo mundo. En segundo lugar, para que esta perspectiva pueda ser considerada como racional y posibilite la sabiduría es preciso que tal perspectiva se reconozca a sí misma de modo auto-reflexivo como tal, es decir como un conocimiento parcial y confuso de una realidad con sentido.
\end{abstract}

PALABRAS CLAVE: Cuerpo; ilustración; Leibniz; perspectivismo; ponto de vista.

\begin{abstract}
This article is intended to analyze the perspectivistic conception of knowledge, as Leibniz presents it in his New Essays, in order to show that this conception is one of the aspects that make the originality of this philosopher in rationalistic Enlightenment. It shall be argued that this conception is based on two theses, the first of which is that Leibniz claims that the human knowledge is essentially perspectivistic, though this does not prevents him of claiming, against skepticism, that in each case the different perspectives present a different piece of knowledge of one and the same world. The second thesis is that, in order to this perspective to be able to be considered as rational and to provide wisdom, it is required that it recognizes itself as such in a reflexive way, i.e., as a partial and confused knowledge of a meaningful reality.
\end{abstract}

KEYWORDS: Body; Enlightment; Leibniz; perspectivism; point of view.

\section{Introducción}

Uno de los rasgos principales de la filosofía contemporánea en su confrontación con la tradición ilustrada consiste en la reivindicación de la facticidad del saber, que no puede ser comprendido correctamente si se pretende reconstruir su naturaleza y su potencialidad mediante una abstracción de las 
determinaciones que lo posibilitan como una perspectiva del mundo situada in concreto. Desde luego, encontramos disparidad de posiciones sobre cómo hemos de entender esta determinación o dar cuenta de los diferentes niveles de originalidad de aquello que constituye nuestro modo de obrar y de conocer. No nos centraremos aquí en las diferentes alternativas dentro de este panorama. Lo importante es señalar que uno de los rasgos principales del pensamiento contemporáneo consiste en el reconocimiento de que sólo es posible dar cuenta del saber en la medida en que no soslayemos que la realidad humana se encuentra esencialmente situada en un borizonte de sentido que condiciona o determina la constitución y cualquier desarrollo particular del saber mismo en cualesquiera de sus expresiones. Por este motivo, se descarta por principio cualquier proyecto de explicación, fundamentación y crítica del saber humano que pretenda soslayar el reconocimiento del valor determinante de esta situación original, mediante la pretensión de llevar a cabo una abstracción de la misma. Pues toda crítica se encuentra ya situada en una determinada perspectiva.

Esta visión se ha construido principalmente como reacción al proyecto ilustrado, especialmente al criticismo kantiano. Kant, al atender a la mera formalidad de nuestro ser sensible, abstrayó por completo de cualquier determinación de lo sensible que sea debida meramente a la individualidad del sujeto. Lo individual no es independiente de toda experiencia, y por tanto carece 
de cualquier valor en la constitución ontológica y cognoscitiva de lo real. ${ }^{2}$ Puede considerarse que el proyecto de la Ilustración racionalista o criticista queda marcado en general por la pretensión de lograr una depuración en la razón tanto a nivel teórico como práctico. En general, desde Descartes a Kant, la fundamentación del saber sólo puede lograrse tras llevar a cabo una labor filosófica de tipo propedéutico que, sirviéndose del método o de la crítica, logre depurar al sujeto que conoce de aquellas determinaciones que puedan obstaculizar la consecución del conocimiento auténtico y fundado. Pero en la Ilustración racionalista podemos hallar también una concepción alternativa, según la cual una comprensión de la realidad humana no debe en absoluto soslayar que el ser humano se encuentra determinado por su relación con una realidad más amplia que le sirve de horizonte, y en relación con la cual se sitúa como una perspectiva parcial y singular. Esta posición no supone en sí misma un abandono del interés por la fundamentación, la corrección y el desarrollo del saber, así como por la emancipación y el progreso de la realidad humana en el ámbito práctico. Se trata de una descripción puede corresponderle a uno de los filósofos clave para la génesis de la Ilustración alemana, Gottfried Wilhelm

${ }^{2}$ No podemos considerar por tanto que Kant desarrolle el perspectivismo leibniziano meramente a partir del argumento de que su fundamentación del conocimiento parte del reconocimiento de la sensibilidad humana, tal como sostiene Holze (2001, pp.519-520). En cierto respecto, Kant radicaliza el perspectivismo leibniziano, en la medida en que reconoce la dependencia discursiva del entendimiento con respecto a la sensibilidad, pero, a diferencia de Leibniz, juzga que una fundamentación de la posibilidad del conocimiento puede y debe pasar por un aislamiento de la formalidad de lo sensible, es decir por una abstracción con respecto a todo aquello que no es completamente independiente de toda experiencia, de tal modo que la diversidad individual de los sujetos no es en absoluto reconocida como constitutiva del conocimiento. Sobre la influencia de Leibniz y el leibnizianismo en Kant, véase Sánchez Rodríguez (2016). 
Leibniz. $^{3}$

En este artículo comprobaremos que en su pensamiento, tal como es expuesto en los NE, existe un claro reconocimiento de la naturaleza esencialmente perspectivista del saber. A la base de su pensamiento metafísico encontramos una concepción que nos obliga a atender de modo simultáneo tanto al concepto de la individualidad como al concepto de la totalidad que lo comprende. Sólo por tal relación de cada individuo con respecto a la totalidad en la que se inscribe puede éste ser entendido como una perspectiva diferente y autónoma que versa sobre un mismo mundo.

Con el objeto de ilustrar esta interpretación, en primer lugar nos centraremos en la teoría de la percepción y la corporeidad en Leibniz. En segundo lugar, se expondrá en qué sentido leemos esta teoría como una concepción perspectiva del conocimiento. Finalmente, se defenderá que esta concepción es coherente con la teoría leibniziana de la sabiduría.

\section{El cuerpo y las pequeñas percepciones insensibles}

Una de las peculiaridades que primeramente llama la atención de los NE es el peso que Leibniz le otorga al concepto de las pequeñas percepciones insensibles. De hecho, es el tema principal del prefacio a la obra, lo cual puede resultar evidente desde que se observa que el reconocimiento de que existen percepciones insensibles o inconscientes le permite a Leibniz presentar una

${ }^{3}$ Sobre la relación entre la concepción perspectivista de Leibniz y el concepto de interpretación en la hermenéutica de Leibniz, véase Nicolás (2016). 
objeción fundamental, que hará valer contra Locke en las diferentes secciones a través de sus fructíferas derivaciones. Ahora bien, a la hora de ir presentando sus diferencias con respecto a Locke mediante el empleo de este concepto, en el contexto de su discusión sobre el carácter innato de las ideas, las cualidades secundarias o la identidad personal, Leibniz no sólo está mostrando las insuficiencias del Essay en relación con tales problemas, sino que a la vez está desarrollando de modo coherente sus propias ideas y concepciones sobre la base de este concepto.

De hecho, es patente que en el prefacio Leibniz defiende que el concepto de pequeñas percepciones es tan fructífero que es posible entender a partir del mismo otros principios fundamentales de su filosofía. De cualquier modo, ya sabemos que Leibniz se sirve de los principios de su sistema tanto para convertirlos en puntos de partida como en puntos de llegada, que han de entenderse a su vez a partir de otros principios. De hecho, la relevancia del concepto de pequeña percepción insensible en los $N E$ viene dada por la conexión sistemática de este concepto con respecto a principios fundamentales de su pensamiento, como la hipótesis de la armonía preestablecida o el principio de continuidad.

Las pequeñas percepciones insensibles son estados subjetivos de la mónada espiritual, pero éstas pueden representar el mundo en la medida en que la actividad interna de la mónada se produce en conformidad con el cuerpo que le acompaña de modo esencial: "Tous les esprits finis sont tousjours joints à 
quelque corps organiques, et ils se representent les autres corps par rapport au leur. Ainsi leur rapport à l'espace est aussi manifeste que celuy des corps"4. En sí misma, una sustancia espiritual como tal, entendida en sentido metafísico, no puede estar situada en una relación espacial con respecto al mundo. Por este motivo, ésta sólo puede representar los cuerpos que componen el mundo en la medida en que se encuentra en una relación determinada con su propio cuerpo, porque este cuerpo a su vez expresa una relación con el resto de los otros cuerpos. Por este motivo, la mónada sólo puede representar los otros cuerpos en el espacio por mediación de su relación metafísica con su propio cuerpo, el cual introduce la determinación de esta mónada como una perspectiva particular sobre el mundo. Si la mente puede llegar a ser consciente de sus percepciones y de la relación que éstas expresan con el mundo al que se refieren es porque previamente esta mente se encuentra originalmente determinada en conformidad armónica con un cuerpo que marca la posición aparente de la misma en el espacio y en el tiempo, y por lo tanto como una determinada perspectiva o relación con el resto de los cuerpos. A este respecto, las ideas no pueden ser meras formas o modos del pensamiento, sino más bien disposiciones que no pueden ser entendidas como facultades puras, sino más bien como “restes des impressions passées dans l'ame aussi bien que dans le corps, mais

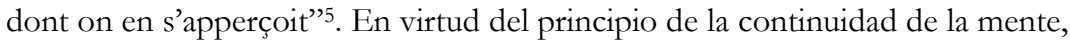

${ }^{4} \mathrm{NE}, \mathrm{A}$ VI 6, p.155; véase también Monadologie § 62; GP VI, p.617. Sobre la relación entre corporeidad y perspectiva en Leibniz, véase Nicolás (2013).

${ }^{5} \mathrm{NE}, \mathrm{A}$ VI 6, p.140. 
toda idea es expresión de percepciones previas del alma, y mediante el principio de la armonía entre el alma y el cuerpo, todo estado perceptivo previo es traducción subjetiva de una traza radicada en la corporeidad.

Por lo tanto, las pequeñas percepciones constituyen en Leibniz, como en Descartes y Kant, el fondo en el que residen las sensaciones y las pasiones, pero para el primero, a diferencia de estos pensadores, la construcción de una teoría de la racionalidad no pasa en absoluto por la exigencia de depuración de tales contenidos, que inicialmente permanecerían en la oscuridad, como tampoco por el aislamiento de lo que podemos conocer a priori sobre la realidad a través del entendimiento puro, que abstrae por completo de las determinaciones particulares de cada sujeto cognoscente. A este respecto, si el alma puede representar el mundo es sólo en la medida en que ésta de modo originario expresa ya, por sí misma misma y por su ser individual y finito, el cuerpo que esencialmente le pertenece: "Car l'ame es un petit monde où les idées distinctes sont une representation de Dieu et où les confuses sont une representation de l'univers."

El concepto de expresión es fundamental para comprender el carácter perspectivista de la mónada, pues ésta no representa el cuerpo y con ello el mundo de un modo figurativo; más bien, toda representación es posibilitada en la medida en que entre los dos polos del espacio de la representación se presupone más bien una relación de expresión: es decir, una relación reglada de 
tipo sistemático entre ambos, "puisqu'il y a un certain rapport exact et naturel entre ce qui es projetté et la projection qui s'en fait, chaque point de l'un repondant suivant une certaine relation à chaque point de l'autre."’7 El alma no es una copia figurativa del cuerpo a través de sus representaciones, pero entre ambos debe presuponerse una relación analógica reglada, según la cual sí existe una correspondencia entre lo que se proyecta y la proyección que de ello resulta. El alma se proyecta a sí misma a través del despliegue y la aclaración de sus percepciones, pero si el resultado de este proceso activo interno a la mónada puede constituir un sistema de conocimientos distintos y objetivos, ello se debe a que previamente la sustancia espiritual presenta originalmente una conformación ordenada según una regla o razón que le es común a ella y a su cuerpo. De este modo, la hipótesis de la armonía preestablecida entre el alma y cuerpo, junto con la aplicación del principio de continuidad a la explicación de la relación entre las representaciones del alma, debe ser interpretada como una condición trascendental que hemos de suponer a priori para explicar la posibilidad de que la representación subjetiva de la mónada sea a la vez una representación adecuada y objetiva de lo real.

A este respecto, la consecución del conocimiento distinto no se produce en la medida en que se ha demostrado previamente desde la razón que esta misma razón no nos engaña en relación con las ideas que es capaz de pensar con evidencia, tal como pretendía Descartes. Mientras que Descartes sí plantea 
la falacia de una razón filosófica que examina la falibilidad original de la razón natural y pretende alcanzar un punto de vista completamente indeterminado, Leibniz juzga que cualquier indagación por parte de la razón sólo es posible en la medida en que se presupone a priori que esta razón que pretende criticar su propia validez y dudar sobre la verdad de sus creencias sólo puede emprender tal empresa desde una perspectiva que presupone precisamente aquello que pretende ser demostrado. No es lo mismo errar que ignorar, ${ }^{8}$ y en este reconocimiento de la finitud de la capacidad natural del conocimiento humano ${ }^{9}$ radica la disociación entre verdad y evidencia que encontramos a la base de la revisión por parte de Leibniz de la teoría cartesiana de los tipos de conocimiento. De la misma forma que es posible que el mal coexista con un Dios justo, también debemos seguir admitiendo que la posibilidad del error coexista con la racionalidad humana, precisamente en la medida en que lo expresado confusamente por la mónada espiritual a través de sus pequeñas percepciones se corresponde con la verdad. ${ }^{10}$ Así, no sólo contra Descartes, sino también contra Locke, Leibniz sostiene que existe una correspondencia entre lo aperceptible y la verdad, la cual se conserva precisamente mediante las percepciones insensibles. ${ }^{11}$

Por lo tanto, el planteamiento de Leibniz no pretende en absoluto emprender un proyecto de fundamentación de la razón sobre la base de un 
aislamiento de sus condiciones a priori que abstraiga de las determinaciones que constituyen el ser mismo de la razón. La racionalidad humana sólo es tal en la medida en que se encuentra situada como una determinada perspectiva que se corresponde de modo fundado con la realidad. La adecuación entre razón y realidad no es un objeto para la reflexión filosófica, desde el momento en que esta última descubre que sólo podemos plantearnos con sentido esta cuestión bajo el presupuesto de que esta conformidad es ya efectiva, pues sólo se puede dar cuenta de la posibilidad de la razón si se admite que su fundamento último es conforme con el fundamento último de la realidad.

Por este motivo, la racionalidad sólo puede emprender un conocimiento distinto y reflexivo de lo real en la medida en que este conocimiento es entendido como el desarrollo continuo, desde una determinada perspectiva, de la totalidad que se encuentra virtual o confusamente conocida ya en y desde esta perspectiva. A este respecto, la individualidad sólo es tal en la medida en que se define y constituye por estar ya situada y determinada en virtud de la posición en la que se inserta en relación intrínseca con una totalidad. A la vez, la totalidad no es manifiesta a la razón humana por ser conocida de modo distinto, sino por aportar una verdad presupuesta de modo confuso en el ser mismo de la razón individual.

La oposición de Leibniz a Locke con ocasión de la suposición del último de que la mente es una tabula rasa e indeterminada permite ilustrar con claridad esta diferencia del primero, no sólo con el mismo Locke, sino con la mayoría de los filósofos ilustrados que, a través de ficciones teóricas han 
pretendido hablar de la mente humana como de un facultad pura e indeterminada. Para Leibniz, tal visión de la racionalidad sólo puede derivarse de nociones incompletas, es decir, de abstracciones que buscan justificar la génesis, el alcance y la validez del conocimiento tras haber despojado a la razón precisamente de aquello que se trata de justificar.

Por este motivo, la defensa de la validez de la razón no puede construirse sobre una abstracción que deje de reconocer el carácter individual, limitado y finito de cualquier desarrollo de la racionalidad humana. Leibniz vuelve a recurrir al concepto de pequeñas percepciones y a su dependencia con respecto al cuerpo para justificar la vigencia del principio de la identidad de los indiscernibles:

Cette connoisance des perceptions insensibles sert aussi à expliquer pourquoy et comment deux ames humaines, ou autrement d'une même espece, en sortent jamais parfaitement sembables des mains du Createur, et ont toujours chacune son rapport originaire aux points de veuë, qu'elles auront dans l'univers. ${ }^{12}$

Sólo podemos hablar de la razón del individuo como la razón que se despliega desde una determinada perspectiva, pues:

Les choses uniformes et qui en re[n] fermet aucune varieté, en sont jamais que des abstractions [...]. Et selon les demonstrations que je crois avoir, toute chose substantielle, soit ame, ou corps, a son rapport à chacune des autres qui luy est propre [...]. Pour ne par dire que ceux qui parlent tant de cette Table rase aprés luy avoir osté les idées, en sauroient dire ce qui luy reste $[\ldots]^{13}$ 
Por ello, la crítica a la concepción lockeana de la mente como una tabura rasa no se limita a sostener que disponemos de los conocimientos en nosotros mismos y nos encontramos determinados por ellos, sino que por ellos también nos encontramos determinados de diversas maneras, pues "[...] oú trouvera-t-on des tablettes qui en soient quelque chose de varié par elles mêmes?" ${ }^{14}$. Son las pequeñas percepciones, como expresión confusa de las impresiones que los cuerpos ejercen sobre nuestro propio cuerpo, las que expresan "cette liaison que chaque estre a avec tout le reste de l'universe"15. Así, cada razón individual expresa un mismo mundo de modos diferentes en la medida en que significa una perspectiva diferente de una misma realidad.

Por lo tanto, cada mónada expresa de modo intrínseco e individual su relación con el resto de las sustancias ${ }^{16}$ por mediación de las pequeñas percepciones insensibles, debido a la relación esencial de las mismas con el resto del universo. Pero, a la vez, el conjunto de estas percepciones no puede ser entendido como una totalidad estática, pues cada percepción aislada se encuentra a su vez en una relación continua y reglada con el resto de las percepciones que conforman esta totalidad subjetiva, pero no meramente en un sentido sincrónico y actual, sino diacrónico e histórico, por la misma regla de continuidad que explica la relación singular de la mónada con el resto de los

\footnotetext{
${ }^{14} \mathrm{NE}, \mathrm{A}$ VI 6, p.53.

${ }^{15} \mathrm{NE}, \mathrm{A}$ VI 6, p.55.

${ }_{16}^{16}$ Para Leibniz, la naturaleza enjuiciada de modo mecánico puede ser pensada como un universo de mónadas, en conformidad con su armonía máximal, según la cual "existe la máximamente posible plenitud de esencia", lo cual sustenta a su vez la suposición de que los cuerpos son informados por un espíritu. Véase al respecto Busche (1997, pp.522-523).
} 
individuos. Así, cada estado actual de la mónada debe ser entendido como la huella o expresión de sus estados precedentes, y a la vez como anticipación confusa de sus estados futuros:

Ces perceptions insensibles marquent encore et constituent le même individu, qui est caracterisé par les traces, qu'elles conservent des estats précedens de cet individu, en faisant la connexion avec son estat present, qui se peuvent connoistre par un esprit superieur, quand cet individu même ne les sentiroit pas, c'est à dire lorsque le souvenir exprés n'y seroit plus. ${ }^{17}$

Por lo tanto, el individuo no sólo significa una perspectiva o un determinado punto de vista insertos en el universo, sino a la vez y principalmente una perspectiva inserta en su propia historia o, más bien, el individuo significa una perspectiva situada en el despliegue diacrónico del universo en la medida en que significa una perspectiva situada en su propia historia individual, que lo constituye como tal.

\section{Conocimiento necesario y perspectiva}

Desde el planteamiento que he tratado de reconstruir puede comprenderse que Leibniz no explique la posibilidad del conocimiento como el resultado de ejercer una abstracción destinada a superar la determinación aportada por esta relación perspectivista del individuo con respecto a la totalidad. El papel que desempeña el concepto de pequeñas percepciones insensibles en esta concepción es inseparable de la aplicación del principio de 
continuidad. Bajo el supuesto de la continuidad de las percepciones que conforman la mente, Leibniz entiende el desarrollo del conocimiento distinto y reflexivo como un despliegue del horizonte de sentido previo que aporta la multiplicidad de las pequeñas percepciones. Este horizonte ofrece la determinación en que se asienta el punto de vista desde el que se despliega la relación original y fundamental entre individualidad y totalidad, presupuesto fundamental para el conocimiento. Para entender el carácter perspectivista de la mónada espiritual hemos de considerarla como un punto de carácter representativo y expresivo, pero a la vez como un punto que se proyecta a sí mismo hacia una totalidad, a la que apunta en su despliegue interno y según un fundamento compartido con esta totalidad.

Por este motivo, podemos considerar que el desarrollo individual de la mónada es el desarrollo reflexivo de un conocimiento previo de la realidad objetiva e intersubjetiva, pero siempre de un modo diverso, situado y determinado. Mientras que para un filósofo como Kant la crítica ha de ejercer una separación de los elementos a priori y a posteriori tanto de la sensibilidad como del entendimiento, por considerar que lo a posteriori no contiene más que la marca de la parcialidad individual, para Leibniz esta parcialidad individual pasa a convertirse en un verdadero a priori de la razón, en la medida en que el conocimiento se forma gradualmente como un desarrollo del fondo oscuro del alma, en el caso de las verdades de hecho, o es descubierto con ocasión del movimiento y la acción de las percepciones que forman este fondo, en el caso de las verdades de razón: 
Et tout cela fait bien juger, que les perceptions remarquables viennent par degrés de celles, qui son trop petites pour estre remarquées. En juger autrement c'est peu connoitre l'immense subtilité des choses, qui enveloppe toujours et partout un infini actuel. ${ }^{18}$

Ciertamente, Leibniz diferencia con claridad el estatuto de las verdades necesarias así como de las verdades abstractas, cuya fuente se encuentra plenamente en el entendimiento, ${ }^{19}$ pero a la vez sostiene que este conocimiento sólo puede ser descubierto en el alma con ocasión del conocimiento sensible: "Car c'est par une admirable Oeconomie de la nature, que nous ne saurions avoir des pensées abstraites, qui n'ayent point besoin de quelque chose de sensible [...]. Mais cela n'empeche point que l'esprit ne prenne les verités necessaires de chez soy" ${ }^{20}$ A la vez, la diversidad individual de cada perspectiva permite entender "que dans cette multitude de nos connoissances nous soyons determinés par quelque chose à renouveller l'une plûtot que l'autre, puisqu'il est impossible de penser disctinctment tout à la fois à tout ce que nous savons"21:

$[\ldots]$ car il est incontestable que les sens ne suffisent pas pour en faire voir la necessité: et qu'ainsi l'esprit a une disposition (tant active que passive) pour les tirer lui même de son fonds; quoique les sens soient necessaries pour luy donner de l'occasion et de l'attention pour cela, et pour le porter plûtôt aux unes qu'aux autres. ${ }^{22}$

En relación con el conocimiento metafísico, Leibniz juzga que los

${ }^{18 N E, ~ A ~ V I ~ 6, ~ p .56 ~ s s . ; ~ v e ́ a s e ~ t a m b i e ́ n ~} 74$ ss., 81.

${ }^{19} \mathrm{NE}, \mathrm{A}$ VI 6, p.75.

${ }^{20} \mathrm{NE}, \mathrm{A}$ VI 6, p.77.

${ }^{21} \mathrm{NE}, \mathrm{A}$ VI 6, p.77.

${ }^{22} \mathrm{NE}, \mathrm{A}$ VI 6, p.80. 
conocimientos fundamentales sobre el ser de la realidad no son conocimientos que el ser humano haya de descubrir a partir de la aplicación de un método formal o que pueda construir de modo doctrinal tras la crítica previa de las posibilidades de la razón. Las categorías fundamentales de la metafísica son accesibles a la reflexión en la medida en que "nous sommes innés à nous mêmes pour ainsi dire, et qu'il y a en nous: Estre, Unité, Substance, Durée, Changement, Action, Perception, Plaisir, et mille autres objects de nos idées intellectuelles"23. Estas ideas pueden llegar a ser manifiestas de modo distinto a nuestro espíritu en la medida en que previamente surgen de nuestro espíritu, pues no podríamos explicar “comment nous pourrions avoir l'idée de l'étre, si nous n'étions des Étrees nous mêmes, et ne trouvions ainsi l'étre en nous." ${ }^{24}$ Lo mismo puede pensarse de la idea de sustancia, pues "la reflexion suffit pour trouver l'idee de la substance en nous mêmes, qui sommes des substances" 25 . Es cierto que la metafísica sólo es posible para esa sustancia espiritual que, además de representar la totalidad del universo a través de sus propias representaciones, puede apercibirse de las mismas de modo reflexivo y alcanzar un conocimiento distinto de las categorías de la metafísica. Pero esto también significa que esta sustancia espiritual sólo puede reflexionar sobre el ser y la sustancia por ser ella esencialmente ser y sustancia que se pregunta a sí misma y de modo autoconsciente por lo que ella es de modo originario.

${ }^{23} \mathrm{NE}, \mathrm{A}$ VI 6, p.51. 


\section{E1 reconocimiento del carácter perspectivista de la razón como}

\section{condición para la sabiduría}

Ya hemos comprobado que para Leibniz no tiene sentido emprender una indagación sobre la posibilidad del conocimiento humano que parta de una concepción que abstraiga de la circunstancia esencial de que la razón humana, además de ser finita, se encuentra ya determinada y situada en una perspectiva, por su relación con el resto del universo en el que se inscribe tal perspectiva. Tanto en la Théodicée como en los NE es patente que Leibniz sostiene que el reconocimiento reflexivo de la propia finitud es condición indispensable para alcanzar la verdadera sabiduría. En la Théodicée defiende que para poder conocer la inmensidad de Dios es preciso reconocer previamente:

$[\ldots] c$ 'est reconnaitre cependant, avant que de voir que Dieu fait tout le mieux qu'il est possible, suivant la sagesse infinie qui regle ses actions. Il est vray que nous en avons déja des preuves et des essais devant nos yeux, lorsque nous voyons quelque chose d'entier, quelque Tout accompli en soy, et isolé, pour ainsi dire, parmy les ouvrages de Dieu. Un tel Tout, formé, pour ainsi dire, de la main de Dieu, est une plante, un animal, un homme. Nous ne saurions assés admirer la beauté et l'artifice de sa structure. Mais lorsque nous voyons quelque os cassé, quelque morceau de chair des animaux, quelque brin d'une plante, il n'y paroit que du desordre, à moins qu'un excellent Anatomiste ne le regarde: et celuy là même n'y reconnoitroit rien, s'il n'avoit vu auparavant des morceaux semblables attachés à leur tout. Il en est de même du gouvernement de Dieu: ce que nous en pouvons voir jusqu'icy, n'est pas un assés gros morceau, pour y reconnoitre la beauté et l'ordre du tout. ${ }^{26}$

Para Leibniz, el individuo debe reflexionar sobre la visión que le ofrece

${ }^{26}$ Theodicée, GP VI, p.188. 
su perspectiva particular, marcada por la confusión y las apariencias, y reconocerse a sí mismo como una perspectiva parcial e incompleta. ${ }^{27}$ De lo contrario, la visión aportada desde este punto de vista no se reconocería como lo que es, por su relación con una totalidad que nunca puede darse de modo claro y distinto, a saber: una visión parcial cuyo sentido último sólo puede ser justificado por su relación con una totalidad que tan sólo se muestra parcialmente, pero nunca en su totalidad. La propia imagen que se presenta en su aislamiento y desconexión a un mismo punto de vista puede ser interpretada por éste de dos modos diferentes: como una representación absoluta de lo real, y por lo tanto como prueba de la falta de sentido de la realidad, en la que cualquiera de sus partes puede carecer de todo sentido y de conformidad con el resto; o como una representación parcial que, si bien en apariencia puede presentarse como carente de sentido, puede adquirir significado en la medida en que se la interprete como la presentación parcial y limitada de una de las partes que constituye una totalidad ordenada, a partir de la cual esta imagen cobra sentido, pero que no puede ser desvelada por completo. Ahora bien, lo segundo sólo es posible para el punto de vista que se reconoce a sí mismo como tal, como un punto de vista espacio-temporalmente situado y finito. No podemos adoptar el punto de vista de una razón que no sea la humana, por la cual podríamos aportar una explicación perfecta o adecuada del fenómeno. Es cierto que esta razón sólo puede tener un conocimiento confuso e imperfecto de lo real; pero

27Véase al respecto Ferro 2010: 30-32, así como Sánchez Rodríguez (2011). Sobre la relación entre perspectiva, autoconciencia y sabiduría, véase Busche (1997, pp.550-559). 
a la vez esta razón finita tiene la capacidad de la auto-reflexión crítica, por la cual no se limita tan sólo a desplegar una visión parcial de lo real, sino que es capaz de reflexionar e interpretarse a sí misma como una perspectiva. De este modo, la determinación de la mente como una particular perspectiva de lo real es una condición trascendental de la racionalidad sólo en tanto que esta mente, a través de la apercepción, se reconoce a sí misma de modo reflexivo como tal perspectiva. Esta exigencia crítica permite evitar que interpretemos como una verdad absoluta lo que en realidad es una apariencia, la apariencia parcial, singular y velada de una totalidad que se le presenta a una perspectiva racional. A este respecto, el conocimiento sólo es posible a través de abstracciones, pero bajo la condición de “[...] qu'on sache, que ce, qu’on dissimule, y est”"28 La razón humana no está capacitada para desentrañar a priori las razones que subyacen a cada una de las experiencias que se le muestran desde una determinada perspectiva. Para poder interpretar que nada es sin razón, la racionalidad ha de adoptar un punto de vista reflexivo dirigido a su propio punto de vista, por el cual reconozca este último como finito y parcial, pero a la vez lo interprete como integrable idealmente en una totalidad infinita fundada en razón:

Mais il n'appartient qu'à la supreme raison, à qui rien n'echappe, de comprendre distinctement tout l'infini, toutes les raisons et totues les suites. Tout ce que nous pouvons sur les infinités, c'est de les connoitre confusément, et de savoir au moins distinctement, qu'elles y sont; autremnt nous jugeons fort mal de la beauté et de la grandeur de l'univers $[. . .]^{29}$

${ }^{28} \mathrm{NE}, \mathrm{A}$ VI 6, p.57.

${ }^{29} \mathrm{NE}, \mathrm{A}$ VI 6, p. 57. 
Debido a la infinitud de la realidad, sólo podemos tener un conocimiento incompleto e inadecuado de las verdades de hecho; pero no obstante sí podemos conocer que nada es sin razón, bajo la condición de que podamos interpretar reflexivamente que nuestro desconocimiento de "toutes les raisons et toutes les suites" 30 no se deriva de la falta de sentido de la realidad, sino más bien de que nuestra perspectiva sólo nos muestra una visión parcial e incompleta de la misma. ${ }^{31}$

Resumiendo, y a modo de conclusión, dos son las condiciones principales que deben tenerse en cuenta en relación con la concepción perspectivista del conocimiento en Leibniz. En primer lugar, para Leibniz la racionalidad humana debe ser entendida como esencialmente perspectivista, lo cual explica no sólo que todo conocimiento se le presente al individuo como un punto de vista parcial y confuso, sino también que cada punto de vista, a pesar de su diversidad, ofrezca un conocimiento objetivo de una misma realidad. En segundo lugar, para que esta perspectiva pueda ser considerada como racional y posibilite la sabiduría es preciso que tal perspectiva se reconozca a sí misma de modo auto-reflexivo como tal, es decir como un conocimiento parcial y confuso de una realidad con sentido. De lo contrario, nos encontraríamos ante un punto de vista parcial y finito que, al interpretarse a sí mismo como absoluto, creería estar ante un conocimiento cierto de la falta de sentido de la realidad cuando lo

\footnotetext{
${ }^{30} \mathrm{NE}, \mathrm{A}$ VI 6, p. 57.

${ }^{31} \mathrm{En}$ la Théodicée también encontramos esta defensa del principio de razón como un principio que sólo puede ser manifiesto para una razón que se reconoce como finita, de tal modo que es capaz de reconocer su propio punto de vista como una perspectiva siempre parcial; véase por ejemplo GP VI, p.197. Sobre esta cuestión, véase Sánchez Rodríguez (2011, pp.9629-66).
} 
que en realidad se le presenta es un conocimiento parcial de la inmensidad de la realidad, cuyo sentido puede en todo caso sobrepasar la racionalidad humana, pero en ningún caso contradecirla.

\section{Ediciones de los escritos de Leibniz}

LEIBNIZ, G. W. Sämtliche Schriften und Briefe. Herausgegeben von der BerlinBranderburgischen Akademie der Wissenschaften und der Akademie der Wissenschaften in Göttingen. Darmstadt, 1923 y sgts., Leipzig, 1938 y sgts., Berlin, 1950 y sgts.

- Die philosophischen Schriften, herausgegeben von C. I.

Gerhardt. 7 Bände, Berlin. 1875-1890.

\section{REFERENCIAS BIBLIOGRÁFICAS}

BUSCHE, Hubertus. Leibniz's Weg ins perspektivische Universum. Eine Harmonie im Zeitalter der Berechnung. Hamburg: Meiner, 1997.

FERRO, Nuno. "El verdadero punto de vista. Sistema y finitud en la hipótesis de la armonía". En SÁNCHEZ RODRÍGUEZ, M. Leibniz en la filosofía y la ciencia modernas. Granada: Comares: 17-32, 2010. 
HOLZE, Erhard. 'Mensch - Perspektive - Gott. Leibniz' Perpsektivitätstheorie als neuzeitliches Pluralismusmodell”. En POSER, H. et alii. Nibil sine ratione. Mensch, Natur und Technik im Wirken von G. W. Leibnir, Hannover: Gottfried-Wilhelm-Leibniz-Gesellschaft, $2^{\mathrm{a}}$ parte, pp.516-523, 2001.

NICOLÁS, Juan Antonio. “Gnoseología del perspectivismo corporal en Leibniz". Cuadernos Salmantinos de Filosofía, 40, 2013, pp. 121-133.

. "Perspective as mediation between interpretations", en Nicolás, Juan Antonio, et alii. Leibniz and Hermeneutics, Cambridge: Cambridge Scholar, 2016, pp. 17-32.

SÁNCHEZ RODRÍGUEZ, Manuel. "Die Erhabenheit des Leidens in den Essais de Théodicée von Leibniz". En: BREGER, Herbert, et alii. IX. Internationaler Leibniz-Kongress, unter der Schrimhersschaft des Bundespräsidenten. Natur und Subjekt. Hannover, 26. September bis 1. Oktober 2011, Hannover: Willhelm-Gottfried-Leibniz-Gesellschaft, 2011, pp. 962-972.

" "La teoría de los tipos de representación en Leibniz y sus principales influencias en la estética y la lógica de la Ilustración alemana”. Cultura. Revisa de História e Teoria das Ideias, 32, pp. 271-294, 2014. 
and Critical Transformation of the Leibnizian-Wolffian Philosophy". En ALTMAN, Matthew, The Palgrave Kant Handbook, London, New York, 2016 (en prensa).

E-mail: msr@ugr.es 